Plans were disseminated to staff by email, in clinical practice meetings and through a poster campaign, including messages empowering staff to say no to interruptions.

Staff were also encouraged to report any errors with the promise that incident reports were solely to be used to highlight systematic issues, and that staff would receive feedback on the outcomes of an incident form.

Measurement of improvement Monthly audits of medication errors were carried out, assessing the number and type of errors occurring, and comparing this to the number of electronic incident reports completed.

Effects of changes The audits showed an increase in the proportion of medication errors reported electronically, with an emphasis placed on blame-free reporting, and positive results obtained from resulting investigations, such as highlighting areas in which extra checks may be useful.

However, despite some improvement, underreporting of errors still continues. Staff have been empowered to prescribe and administer medications without interruption, but it remains difficult to accurately measure the effect of this intervention without a reliable screening and reporting method. The monthly audit process has resulted in fatigue from nursing staff, resulting in unreliable reporting, and therefore its usefulness has become limited - a new approach is currently being planned.

Lessons learnt Junior doctor and nursing involvement in attempting to create a medication safety culture has resulted in greater engagement with the process, highlighting the importance of involving all relevant stakeholders in a change project. However, ongoing projects can result in staff fatigue, so methods need to be considered to maintain momentum.

Message for others Incident reporting can be improved through a targeted programme including appropriate feedback and a "no blame' approach. Successfully implementing this kind of culture change requires a multidisciplinary approach and trainee involvement.

Medication incidents are often related to distraction, but assessing the effect of a project to reduce interruptions requires a reliable method of measuring outcomes.

\section{G526(P) QUALITY IMPROVEMENT PROJECT ON IRON INFUSION THERAPY IN A PAEDIATRIC HAEMODIALYSIS UNIT}

A Nuti, M Pernas, R Krishnan. Paediatric Nephrology Department, Cardiff and Vale University Local Health Board, Cardiff, UK

\subsection{6/archdischild-2015-308599.477}

Context The QI project was conducted at the haemodialysis unit in the paediatric nephrology department at Noah's Ark Children's hospital, Cardiff. Stakeholders involved were the medical and nursing staff at the haemodialysis unit caring for children with chronic kidney disease CKD.

Problem Anaemia is prevalent amongst children with CKD. Iron infusion is administered to such children with chronic anaemia. Children on haemodialysis attending the Children Kidney Centre receive iron infusion if they satisfy the criteria based on haemoglobin and serum ferritin values in accordance with departmental guidelines. This involves measurement of C-reactive protein CRP and serum ferritin prior to iron administration. High iron exposure is detrimental to end organ function and hence warrants regular monitoring in conjunction with CRP, another inflammatory marker.

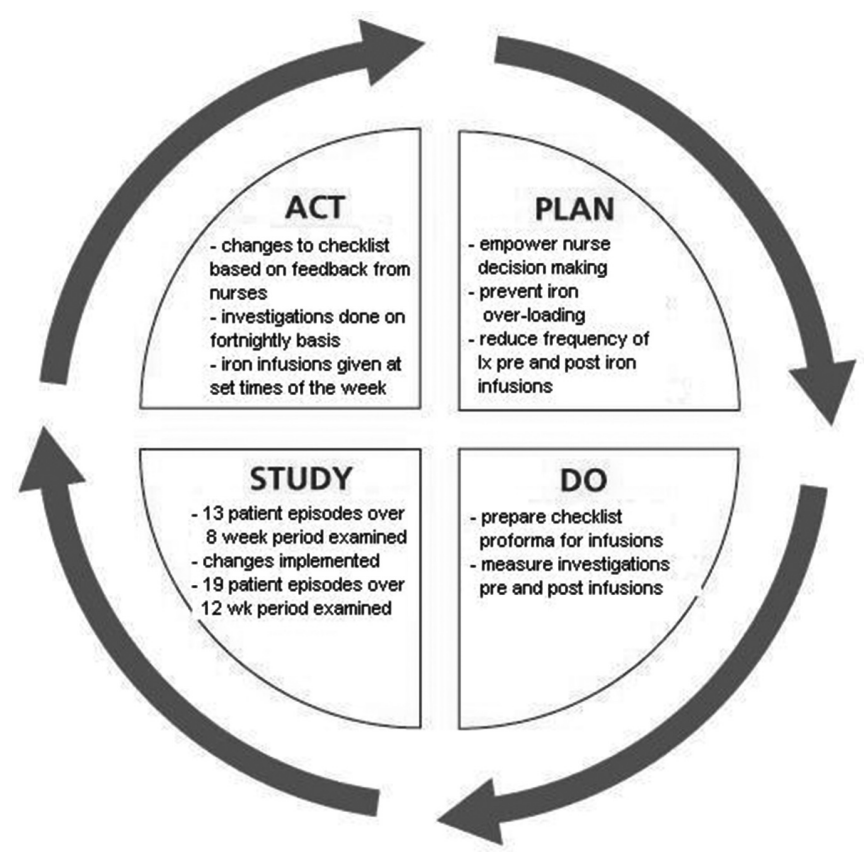

Abstract G526(P) Figure 1

We suspect that some children may be receiving iron infusions despite being iron replete. Also, we may be over investigating these children with anaemia.

Assessment of problem and analysis of its causes We identified all children receiving iron infusion in the haemodialysis unit over an 8 week period. We retrospectively enquired blood investigations done, prior to and after iron infusion. Blood investigations were noted to lag, during pre and post infusion times.

Intervention and Study design We devised a checklist for nursing staff to follow, which looked at set times for measuring haemoglobin, serum ferritin and CRP during the month (at the start of the first and third week of the month) and also tabulating the ferritin values that would trigger frequency of iron infusions. These were aimed to

- prevent iron overloading in patients with chronic anaemia

- regularise the checking of bloods in those receiving iron infusions

- empower the nursing staff to independently take decisions on iron infusion delivery

Strategy for change The following PDSA cycle was employed.

Plan - empower independent decision making on iron infusions by haemodialysis nursing staff

Do - setting up of iron infusion checklist to be followed for every patient who warrants iron infusion for chronic anaemia, with recommendations based on set values of ferritin, CRP and haemoglobin.

Study -analyse adherence to checklist in 3 months time

Act - make appropriate changes to workplace behaviour based on findings of PDSA cycle.

Measurement of improvement The measurement encapsulated all three domains of the Donabedian framework: Structure (investigating chronic anaemia of CKD) + Process (decision to administer iron infusion) $=$ Outcome (blood investigations validity). We analysed 13 patient episodes at the commencement of the project and a total of 19 patient episodes at the end of the improvement cycles. 
Effects of changes Blood investigations done were more regularised and not excessive in comparison from those done previously. Nursing behaviour with regard to initiation and maintenance of iron infusion became more independent. Consequently, it freed up doctor-time and empowered nursing decision making skills. This also resulted in improved team morale and ultimately patient safety by mitigating human errors.

Lessons learnt The checklist was improved based on feedback obtained after the first PDSA cycle. A second cycle showed that investigations done were now optimised. The third cycle showed improved adherence and compliance with prevention of over treatment with iron infusion.

Message for others For any QI project, interventions should be carefully designed. Stakeholder buy-in and easy accessibility of the intervention improves sustainability. Performing multiple PDSA cycles and incorporating the feedback is vital to any QI project.

\section{G527(P) BUCKLE FRACTURES OF THE DISTAL RADIUS: INCREASED EFFICIENCY AND COST SAVINGS THROUGH A NEW MANAGEMENT PATHWAY}

KM Knight, G Hadley, A Parikh. Paediatric Emergency Department, Royal London Hospital, London, UK

\subsection{6/archdischild-2015-308599.478}

Context This quality improvement project took place in a tertiary centre, involving the Paediatric A\&E and Orthopaedic teams. It required minor changes in practice by paediatric A\&E doctors and nurses, and affected children between 3 and 15 years who presented with a radial buckle fracture.

Problem Fractures are a common reason for children to attend A\&E. During the summer months, fracture incidence tends to increase. Our summer fracture clinics were quickly filling up with minor injuries (up to a 3 week wait) and this impacted upon patients with severe injuries who needed urgent orthopaedic review.

Buckle fracture of the distal radius was one type of injury seen with disproportionate frequency.

Assessment of problem and analysis of its causes A joint paediatric/orthopaedic meeting addressed the issue of overbooked clinics. The orthopaedic team noted that many patients with a buckle fracture were discharged with no intervention at their first fracture clinic appointment.

Analysing the previous summer's buckle fractures, over $90 \%$ were managed with a cast, repeat $\mathrm{x}$-ray and fracture clinic review. Our practice was outdated - recent studies advocate conservative management of this injury with a removable splint, and no further review.

Intervention A new management pathway for buckle fractures was created. If a set of criteria were met, the patient could be discharged with a splint rather than a cast.

The criteria were: fracture of radius only; no break in opposite cortex; minimal angulation on AP and lateral X-rays; no child protection concerns.

The emergency department sourced paediatric splints. Parents were educated before leaving, and given an information leaflet. As a 'safety net', all X-ray reports were reviewed by a registrar if the fracture was more serious than first thought, the child was recalled.

Study design All children with suitable fractures during JuneJuly 2014 were managed according to the new guideline. Their records were later analysed and a follow up telephone survey evaluated how the child and family had coped with the splint. Clinic waiting times were monitored.

Strategy for change A draft guideline for buckle fracture management was agreed between paediatric A\&E and orthopaedic doctors, and disseminated by email to all A\&E staff. Posters displayed around the department reminded everyone of the change in practice.

A parent information leaflet was produced in the two common languages of our area.

During an initial two week trial period, staff were encouraged to share their experiences of using the new guideline, which allowed certain details to be amended before the two month study period.

Measurement of improvement Fracture clinic bookings were monitored, and a telephone survey evaluated parental satisfaction with management.

We used electronic records to check whether there were any post fracture problems presenting to $A \& E$, and also calculated how much money and time the department would save using this new pathway.

Effects of changes In two months: 37 fewer fracture clinic appointments were booked, equating to three full clinics' worth of appointments. 22.2 nursing hours were saved as fewer casts were applied. Only two out of 24 fracture clinics that took place were overbooked. There was a $65 \%$ response rate in the telephone survey; all families contacted were very happy with the removable splint. Of those who did not respond, none reattended.

The department saved over $£ 90$ per child. The new guideline is now our standard practice.

Lessons learnt Modernising guidelines in line with latest research may lead to greater efficiency, and significant savings.

Message for others Cooperation between different teams can be the catalyst for effective change.

\section{G528(P) A DAILY REGIONAL CONFERENCE CALL: MAXIMISING EFFICIENCY OF REPATRIATION. THE IMPACT ON NEONATAL UNITS IN THE WEST OF SCOTLAND}

${ }^{1} \mathrm{~J}$ Mitchell, 1,2 L Jackson, ${ }^{2} \mathrm{~J}$ Elliot, ${ }^{2} \mathrm{~F}$ Tait. 'West of Scotland Neonatal Transport Team, NHS Greater Glasgow and Clyde, Glasgow, UK; ${ }^{2}$ West of Scotland Neonatal Managed Clinical Network, West of Scotland Managed Clinical Network, Glasgow, UK

\subsection{6/archdischild-2015-308599.479}

Context This project has been carried out between the regional neonatal units, the Neonatal Managed Clinical Network (NMCN) and the Neonatal Transport team (NTS). It has involved multidisciplinary team work between all the health boards in the region with input from key medical, nursing and network staff.

Problem It became apparent to the NMCN that neonatal cot capacity in the region was frequently highly occupied and under pressure. We wanted to have a proactive method of identifying cot capacity issues early and maximise our repatriation processes whilst identifying any delays. As a trainee representative at the NMCN meetings, I suggested setting up a regional teleconference call to address these issues. A multidisciplinary team were tasked with setting up this call.

Assessment of problem and analysis of its causes The working group contacted key staff within the region's neonatal 